\title{
When do you need a validated assay?
}

\author{
...the fit-for-purpose paradigm is applicable to several different analytical questions."
}

Keywords: bioanalysis $\approx$ regulatory $₫$ validated $\approx$ validation $\approx$ US FDA

As science advances, the questions we need to address in drug-development burgeons. However, resources are limited, and it is necessary to use these resources strategically. Clearly, it is impossible to do all things. Bioanalytical science is no exception to this phenomenon. In the recent past, some areas of scientific endeavor have expanded extensively, such as better characterization of metabolites and the exploitation of biomarkers in drug-development. As measuring these entities in vivo plays a critical role, many questions have arisen about how much method validation is needed, and when.

The issue of metabolites in safety testing has evolved over a number of years and culminated with the publication of the US FDA Metabolites in Safety Testing Guidance in 2008 [101]. This guidance dealt with the evaluation of novel or unique metabolites observed in early human clinical trials, which were not observed in preclinical safety studies. The concern that is addressed is the unknown safety risks of these metabolites and the document provides several strategies to assess the toxicology of these entities. Some of the difficulty with this issue arises in the timing of these events: these metabolites are seen in early clinical trials (hopefully), when drug-development plans and timelines have already been implemented. Hence, backtracking is difficult.

A requisite corollary to the toxicological assessment of these metabolites is their measurement in vivo. In fact, the guidance stipulates that studies need to be conducted for a metabolite (to assess its toxicity) if its exposure is greater than $10 \%$ compared with the parent. This prerequisite raises a number of questions that are difficult to answer. How do you assess metabolite AUC without a validated assay (for a metabolite you haven't seen previously)? How do you validate an assay for this metabolite when no authentic standards are available? Do you need to go back and repeat the trial with a validated assay? Assessing the activity of the metabolite and possibly measuring it in vivo are necessary steps. However, most of these questions boil down to: how much validation is needed and by what stage of development?

The European Bioanalytical Forum has developed a paradigm for addressing this issue [1]. Essentially, the scheme describes the development and use of an analytical method with varying degrees of validation. In cases where the metabolite is known or expected to be toxicologically active, the notion is to use a validated method for the metabolite beginning with preclinical studies and continuing with clinical development. In the case where the activity of the metabolite is unknown, less validated screening and qualified methods may be more appropriate until a determination is made regarding the toxicology of the metabolite. The European Bioanalytical Forum scheme makes very reasonable sense and may be a very valuable tool for industry.

However, from the regulatory perspective, there are two key issues. First is the determination of metabolite activity. Unexpectedly high concentrations of a metabolite or an altogether new metabolite are not especially problematic if it is inactive, because no further characterization is needed. So the decision to develop a fully validated analytical method for the metabolite hinges on the determination of its toxicology (although, you could argue that measuring the metabolite may contribute to the understanding of its metabolism and the fate of its parent molecule). The trigger is the appearance of $10 \%$ or more of the metabolite compared with parent. Although this is a useful rule, care should be paid to the activity of the molecule. For example, $\mathrm{SN}-38$ is the metabolite of irinotecan. Overall, it accounts for less than $10 \%$ of the parent. Yet its activity is 1000-times that of the parent [2]. If one simply followed the guidance rule, SN-38 may not have been measured, although it contributes significantly to the activity (efficacy and toxicity) of this drug.

The second key issue is the overall purpose of the study. In early studies, the extent of method

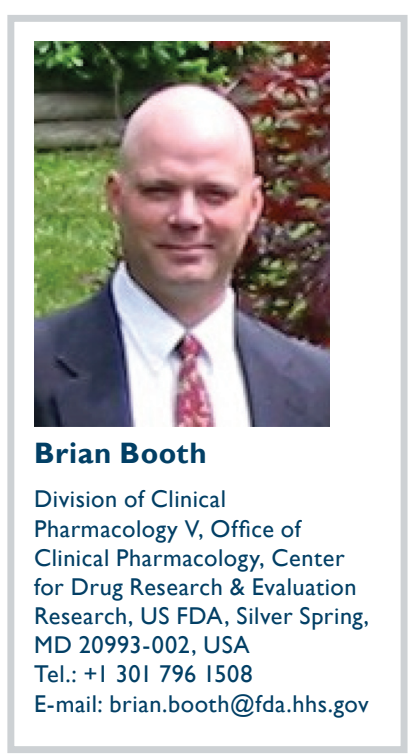

FUTURE
SCIENCE FSS 
validation for an assay of an unexpected metabolite can be relatively low. The regulatory impact of these early studies (e.g., Phase I) is generally limited. Later developmental studies are generally more important in determining the safety and effectiveness of the new drug or biologic, as well as providing the basis for dosing and patient treatment. It is in that setting that the reliability is of greater importance and full method validation is required for the active moieties of the drug. Therefore, a clear understanding of the impact of any given study on the regulatory submission can help to choose an appropriate level of validation.

In a separate, yet related area, the use of biomarkers to assess drug safety and/or effectiveness has increased in recent years. Efforts to quantify these entities have also increased commensurately. But this arena is even more complicated than the 'Metabolites in Safety Testing' situation, because biomarkers can be used a wide variety of ways, which range from preclinical candidate selection, to Phase I proof-of-concept or dose selection, to the evaluation of efficacy in a pivotal trial. The situation can be further complicated by the use of several biomarkers for different purposes at different points of drug-development.

The same question arises in these cases: how well validated does the method need to be for these various biomarker applications? Here again, the answer depends on the purpose of the study being developed.

For instance, if a biomarker is used in a Phase 0 or I trial to assess whether the drug interacts with its target sufficiently in vivo to merit further development, the risk is entirely assumed by the sponsor. Generally, this type of study is quite important to the sponsor in deciding to move forward or not, or to determine what resources to allocate to this drug candidate. However, these studies typically don't contribute significantly to decisions about the overall safety and efficacy of the product. Consequently, the extent of method validation is not of critical regulatory importance. The sponsor can decide what level of analytical uncertainty is acceptable with respect to making their own decision regarding further development of the product.

However, if the biomarker is used in a Phase III trial as the basis for determining efficacy, the reliability of the data is of much greater importance. In this case, a high degree of confidence in the data is needed and the bioanalytical method needs to be fully validated to provide that assurance.
Both of these scenarios are similar to what has traditionally been expected for pharmacokinetic assays of small molecules. The regulatory need is typically greater in later developmental studies, because these data are generally the most important in deciding on approvability, but less so in early development. So there is some latitude deciding on how extensive method validation needs to be. The approach is 'fit-for-purpose'.

In summary, the fit-for-purpose paradigm is applicable to several different analytical questions. The general rule that can be applied is that if the data generated will support regulatory action, such as assessing safety and/or efficacy, or supporting labeled-dosing instructions or patient treatment, then the data must be reliable and the analytical assays should be fully validated. In other cases, where the sponsor will use the data internally to make decisions about candidate selection, or continuing product development, the sponsor can use as much analytical method validation as it deems appropriate to make these decisions.

\section{Disclaimer}

The views expressed in this article are those of the author and do not reflect official policy of the US FDA. No official endorsement by the FDA is intended or should be inferred.

Financial \& competing interests disclosure The author has no relevant affliations or financial involvement with any organization or entity with a financial interest in or financial conflict with the subject matter or materials discussed in the manuscript. This includes employment, consultancies, honoraria, stock ownership or options, expert testimony, grants or patents received or pending, or royalties. No writing assistance was utilized in the production of this manuscript.

\section{References}

1 Timmerman P, Anders Kall M, Gordon B, Laakso S, Freisleben A, Hucker R. Best practices in a tiered approach to metabolite quantification: views and recommendations of the European Bioanalysis Forum. Bioanalysis 2(7), 1185-1194 (2010).

2 Ramchandani RP, Wang Y, Booth BP et al. The role of SN-38 exposure, UGT1A*28 polymorphyism and baseline bilirubin levels in predicting severe irinotecan toxicity. J. Clin. Pharmacol. 47(1), 78-86 (2007).

\section{Website}

101 Guidance for Industry: Safety Testing of Drug Metabolites (2008) www.fda.gov/downloads/Drugs/ GuidanceComplianceRegulatoryInformation/ Guidances/ucm079266.pdf 